\title{
Use of The Velocity of Sound in Predicting the PVT Relations
}

Mohammad R. Riazi( $\left.{ }^{*}\right)$ and G.Ali Mansoori

Department of Chemical Engineering, University of Illinois at Chicago, (Box 4348) Chicago, nllinois 60680, USA

Keywords: theory, equation of state, velocity of sound.

(Received July 17, 1992; accepted in final form April 4, 1993)

\section{ABSTRACT}

Thermodynamic properties of fluids are generally calculated from the PVT relations through equations of state. The majority of existing equations of state require the critical properties or intermolecular potential energy parameters as their input data. In many cases, such properties are neither available nor they can be accurately estimated.

One accessible and accurately measurable property of substances is the velocity of sound. In this report a method is introduced through which one can predict the PVT behavior of fluids using the velocity of sound data. A general mathematical relationship,

$$
c^{2}=c_{h s}^{2}+\frac{-v^{2}}{M} \int_{T}^{\infty}\left(\frac{\partial^{2} T}{\partial v^{2}}\right)_{s}\left(\frac{\partial s}{\partial T}\right)_{v} d T
$$

expressing the velocity of sound, $\mathrm{c}$, in terms of the hard-core velocity of sound, $\mathrm{c}_{\mathrm{hs}}$, and thermodynamic properties is derived. One may use this equation to extract PVT data from cVT data, or vice versa. As an example the virial coefficients, the Lennard-Jones intermolecular potential parameters, and the constants of the van der Waals equation of state for a number of pure fluids are calculated using the velocity of sound data. Utility of this method is particularly attractive for such compounds as heavy hydrocarbons, unstable fluids, and newly designed molecules for which intermolecular parameters and critical properties are not available.

(*) Permanent Address: Chemical Engineering Department, Sharif University of Technology, Azadi Avenue, P.O. Box 11365-9465, Tehran, Iran. 


\section{INTRODUCTION}

Equations of state are generally used to estimate thermodynamic properties of gases, liquids, and solids necessary in design and phase behavior calculations. In most of the equations of state such as the van der Waals, Redlich-Kwong equations, etc., critical properties or intermolecular potential energy parameters are needed as input data to estimate constants in the equations. Properties calculated through equations of state are sensitive to the values of input data used as demonstrated by various investigators (Whitson, 1984; Chorn and Mansoori, 1988). Measured values of critical properties are available for some compounds, but for heavy materials such data cannot accurately be predicted. The main objective of this work is to look at another property, the velocity of sound, which is directly measurable and can be used for evaluation of constants in equations of state instead of critical constants or intermolecular potential energy parameters. Colgate, et al. (1992) have shown how the velocity of sound can be used to predict critical properties. In this work, we show how velocity of sound data can replace the critical constants to estimate the virial coefficients, the van der Waals constants, the Lennard-Jones potential parameters and other equation of state constants. medium is:

The basic thermodynamic relation for the velocity of sound in a $c^{2}=\frac{-v^{2}}{M}\left(\frac{\partial p}{\partial v}\right)_{s}$

where $c$ is the velocity of sound, $v$ is the molar volume and $M$ is the molecular weight. Using thermodynamic property relations (Prausnitz, et al., 1986) it can be shown that eq. 1 is equivalent to:

$c^{2}=\left(-\gamma v^{2} / M\right)(\partial \mathrm{P} / \partial v)_{T}=(\gamma / M)(\partial \mathrm{P} / \partial \rho)_{T}$

in which $\gamma$ is the heat capacities ratio $\left(=C_{p} / C_{v}\right)$ and $\rho$ is the molar density $(=1 / v)$ Considering that the isothermal compressibility, $x_{\mathrm{T}}$, is defined as:

$\kappa_{T}=-\frac{1}{v}\left(\frac{\partial v}{\partial p}\right)_{T}$

The velocity of sound in terms of isothermal compressibility becomes:

$c^{2}=\frac{\gamma}{M \rho \kappa_{T}}$ 
Knowing that the velocity of sound is a state function, an equation of state can be developed for the velocity of sound in terms of temperature and density as the independent thermodynamic variables.

\section{VELOCITY OF SOUND EQUATION OF STATE}

It was shown by Alem and Mansoori (1984) that the expression for the entropy departure of a hard-sphere fluid can be used for entropy departure of a real fluid provided the hard-sphere diameter is taken to be dependent on temperature and density. Using the Carnahan-Starling equation of state for a hard-sphere fluid the entropy departure from the ideal gas can be derived,

$$
\left(s-s^{\mathrm{id}}\right)_{\mathrm{hs}}=-\frac{\mathrm{R} \eta(4-3 \eta)}{(1-\eta)^{2}}
$$

in which $\eta$ is the dimensionless packing fraction defined as:

$\eta=(\pi / 6) \mathrm{N}_{\mathrm{a}} \rho \mathrm{d}^{3}$

$\mathrm{N}_{\mathrm{a}}$ is the Avogadro number, $\mathrm{d}$ is the hard-sphere diameter, and $\rho$ is the molar density. As shown by Alem and Mansoori (1984) for non-associating fluids, $d$, in general, can be taken as a linear function of $1 / T$ and $p$, i.e., $d=d_{0}+d_{1} p+d_{2} / T+d_{3} \rho / T$. From eq. 5 we have:

$s=f(T, v)$

Since $c$ is a state function, we can write:

$\mathrm{c}=\mathrm{c}(\mathrm{s}, \mathrm{v})$

Differentiating eq. 1 with respect to $s$ at constant $v$ gives:

$2 \mathrm{cdc}=\frac{-\mathrm{v}^{2}}{\mathrm{M}}\left(\frac{\partial}{\partial \mathrm{s}}\left[\left(\frac{\partial \mathrm{p}}{\partial \mathrm{v}}\right)_{s}\right]_{\mathrm{v}} \mathrm{Jds}_{\mathrm{d}} \quad\right.$ at constant $\mathrm{v}$

From eq. 7 we have:

$d s=\left(\frac{\partial s}{\partial T}\right)_{v} d T$

at constant $\mathrm{v}$

We also have the following mathematical identity: 
$\frac{\partial}{\partial s}\left[\left(\frac{\partial p}{\partial v}\right)_{s}\right]_{v}=\frac{\partial}{\partial v}\left[\left(\frac{\partial p}{\partial s}\right)_{v}\right]_{s}$

and from the Maxwell relations:

$$
\left(\frac{\partial p}{\partial s}\right)_{v}=-\left(\frac{\partial T}{\partial v}\right)_{s}
$$

Substituting eqs. 10, 11 and 12 into eq. 9 we get:

$\left.2 \mathrm{cdc}=\frac{\mathrm{v}^{2}}{\mathrm{M}} \mid \frac{\partial}{\partial \mathrm{v}}\left[\left(\frac{\partial \mathrm{T}}{\partial \mathrm{v}}\right)_{s}\right]_{\mathrm{s}}\right)\left(\frac{\partial \mathrm{s}}{\partial \mathrm{T}}\right)_{\mathrm{v}} \mathrm{dT} \quad$ at constant $\mathrm{v}$

where $\left(\frac{\partial T}{\partial v}\right)_{s}$ can be determined from eq. 7:

$\left(\frac{\partial \mathrm{T}}{\partial \mathrm{v}}\right)_{s}=-\frac{(\partial \mathrm{s} / \partial \mathrm{v})_{\mathrm{T}}}{(\partial \mathrm{s} / \partial \mathrm{T})_{\mathrm{v}}}$

When $\mathrm{T} \rightarrow \infty$ we have $\mathrm{c} \rightarrow \mathrm{c}_{\mathrm{hs}}$, and integrating eq. 13 gives:

$c^{2}=c_{h s}^{2}+\frac{-v^{2}}{M} \int_{T}^{\infty}\left(\frac{\partial^{2} T}{\partial v^{2}}\right)\left(\frac{\partial s}{\partial T}\right)_{v} d T$

$c_{h s}$ can be estimated from either eq. 1 or eq. 2 using the Carnahan-Starling hard-sphere equation of state. Eq. 15 is a general mathematical relationship for the velocity of sound equation of state which has the following general form:

$c=c(T, v)$

One can use Eq. 15 to extract PVT data from cVT data, or vice versa. Also provided the PVT equation of state for a substance is available one can use Eq. 15 to obtain the parameters of that equation of state from the velocity of sound, cVT data. 


\section{ESTIMATION OF EQUATION OF STATE CONSTANTS FROM THE VELOCITY OF SOUND}

Although eq. 15 can be applied to any PVT relation, here as an example, we use the virial and Carnahan-Starling modified version of the Lennard-Jones equations of state to obtain their constants from velocity of sound data. Assuming equality of the hard-sphere and real fluid entropy departures is valid, the packing fraction of hard-spheres can be calculated from entropy data (eq. 5):

$\left.\eta=(\pi / 6) \rho N_{a} d^{3}=\left\{2-\left(s-s^{i d}\right) / R-\left[4-\left(s-s^{i d}\right) / R\right]^{1 / 2}\right] / 3-\left(s-s^{i d}\right) / R\right\}$

Calculated values of $d$ from eq. 17 in the gas phase indicate the following simpler relation between the hard-core diameter, $d$, and $T$ :

$d=d_{0}+d_{1} / T$

Using the virial equation, truncated after the third term, we get:

$\mathrm{Z}=\frac{\mathrm{pv}}{\mathrm{RT}}=1+\frac{\mathrm{B}(\mathrm{T})}{\mathrm{v}}+\frac{\mathrm{C}(\mathrm{T})}{\mathrm{v}^{2}}$

For a hard-sphere fluid (when $\mathrm{T} \rightarrow \infty$ ) we have:

$Z_{h s}=\lim _{T \rightarrow \infty} Z=1+\frac{B_{h s}}{v}+\frac{C_{h s}}{v^{2}}$

Since entropy departure from the ideal gas reference state of unit pressure is equal to:

s-sid $=\int_{\infty}^{v}\left[\left(\frac{\partial p}{\partial T}\right)_{v}-\frac{R}{v}\right] d v$

by applying eqs. 19 and 20 into eq. 21 we get:

$\left[\frac{s-s^{i d}}{R}\right]_{\text {real fluid }}=-\left\{\ln p+\frac{B+T B^{\prime}}{v}+\frac{C+T C^{\prime}}{2 v^{2}}\right\}$

$\left[\frac{\mathrm{s}-\mathrm{sid}}{\mathrm{R}}\right]_{\text {hard sphere }}=-\left\{\ln \mathrm{p}+\frac{\mathrm{B}_{\mathrm{hs}}}{\mathrm{v}}+\frac{\mathrm{C}_{\mathrm{hs}}}{2 \mathrm{v}^{2}}\right\}$

where $B^{\prime}$ and $C^{\prime}$ are the first order derivatives of $B$ and $C$ with respect to 
temperature. Equating the right-hand-sides of eqs. 22 and 23 results in the following relations:

$\mathrm{TB}^{\prime}+\mathrm{B}=\mathrm{B}_{\mathrm{hs}}$

$\mathrm{TC}^{\prime}+\mathrm{C}=\mathrm{C}_{\mathrm{hs}}$

For a hard-sphere fluid, the Carnahan-Starling equation of state gives the following relations for the second and the third virial coefficients (Walas, 1985):

$B_{h s}=(2 / 3) \pi N_{a} d^{3}$

$C_{h s}=(5 / 18) \pi^{2} \mathrm{~N}_{a}^{2} d^{6}$

Non-homogeneous differential equations 24 and 25 can be solved using eqs. 18, 26, and 27. The final solutions for $B$ and $C$ are in the following forms:

$B(T)=q_{0} \frac{\ln T}{T}+\sum_{n=0}^{3} \frac{p_{n}}{T^{n}}$

$C(T)=q_{1} \frac{\ln T}{T}+\sum_{n=0}^{6} \frac{L_{n}}{T^{n}}$

Parameters $q_{n}, p_{n}$ and $L_{n}$ are defined in Table 1 . As it is shown in Table 1 since parameters $k_{1}, k_{2}, d_{0}$ and $d_{1}$ are all known, only parameters $p_{1}$ and $L_{1}$ are unknown. These may be determined from velocity of sound data. Substituting eq. 19 into eq. 2 would result in the following relation for the velocity of sound:

$c^{2}=\frac{\gamma \mathrm{RT}}{\mathrm{M}}[1+\rho(2 \mathrm{~B}+3 \mathrm{C} \rho)]$

From thermodynamic relations for $C_{p}$ and $C_{v}$ when eq. 19 is used for the PVT relation, the following expressions can be derived for the heat capacities:

$$
\begin{aligned}
& C_{p}=C_{p}{ }_{p}^{i d}-R\left[\frac{T^{2} B^{\prime \prime}}{v}-\frac{\left(B-T B^{\prime}\right)^{2}-C+T C^{\prime}-T^{2} C^{\prime \prime} / 2}{v^{2}}\right] \\
& C_{v}=C_{v}^{i d}-R\left[\frac{2 T B^{\prime}+T^{2} B^{\prime \prime}}{v}-\frac{T C^{\prime \prime}+T^{2} C^{\prime \prime} / 2}{v^{2}}\right]
\end{aligned}
$$

$B^{\prime \prime}$ and $C^{\prime \prime}$ are the second derivatives of $B$ and $C$ with respect to temperature 
which can be obtained by differentiating eqs. 28 and 29.

Using experimental data of the velocity of sound, parameters $p_{1}$ and $L_{1}$ can be determined from eq. 30. From these parameters, complete forms of $B(T)$ and $C(T)$ will be determined from eqs. 28 and 29.

\section{TABLE 1}

Algebraic Expressions of Parameters of Equations $28 \& 29$

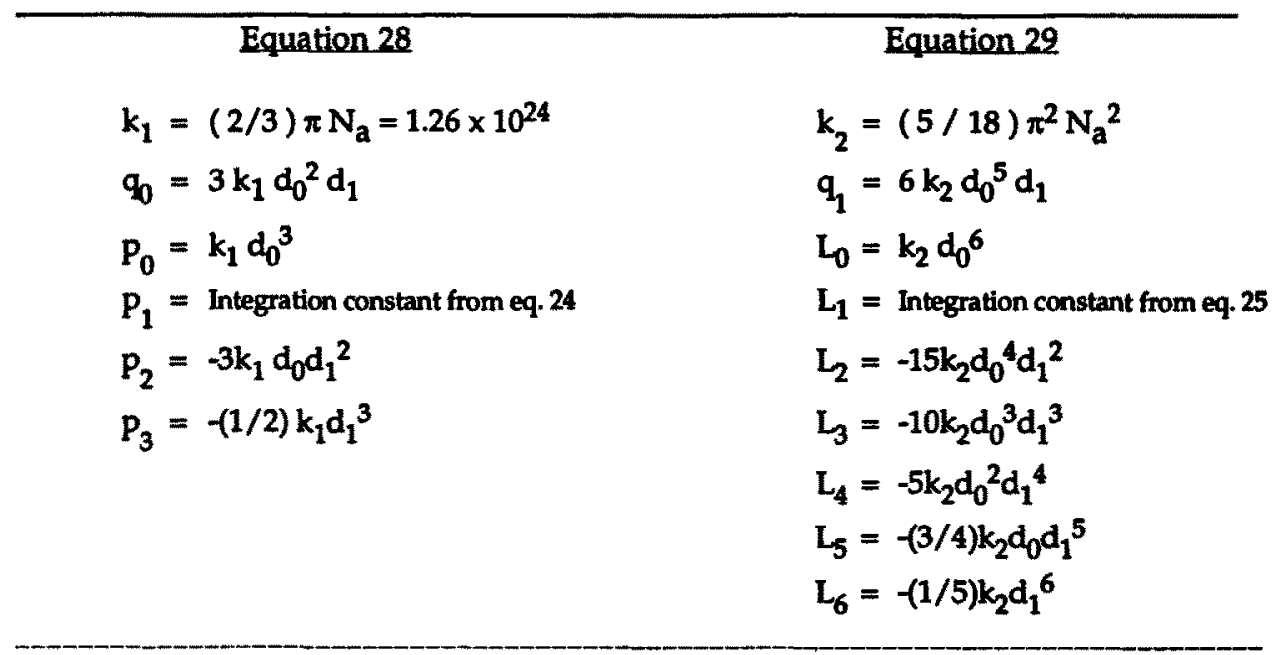

\section{THE LENNARD-JONES CONSTANTS}

The Carnahan-Starling modified form of the Lennard-Jones equation of state is in the following form (Rao and Dutta, 1983; Rao and Gupta, 1985 ):

$\mathrm{p}=\underset{\mathrm{v}}{\mathrm{RT}}\left[\frac{1+\eta+\eta^{2}-\eta^{3}}{(1-\eta)^{3}}-\frac{32 \in \eta}{3 \mathrm{k}_{\mathrm{B}} \mathrm{T}}\right]$

where $k_{B}$ is the Boltzman constant, $\sigma$ is the molecular size parameter and $\epsilon$ is the molecular energy parameter in the Lennard-Jones potential. Here the packing fraction, $\eta$, is defined in terms of parameter $\sigma$ :

$\eta=(\pi / 6) \mathrm{pN}_{\mathrm{a}} \sigma^{3}$

Using eq. 33 for the PVT relation, the velocity of sound, c, can be estimated through eq. 2 in a similar way as for the virial equation of state. 
Eq. 15 can also be applied to any other equation of state such as the van der Waals equation in a similar manner.

\section{RESULTS AND DISCUSSION}

The data for methane as reported by Goodwin (1974) are used to obtain the virial coefficients and the Lennard-Jones potential parameters from the velocity of sound data as discussed in the previous sections. Using the reported data for entropy of methane, the methane hard-core diameter was calculated through eq. 17 . Values of $d_{0}=2.516\left[10^{-10} \mathrm{~m}\right]$ and $d_{1}=554.16\left[10^{-}\right.$ ${ }^{10} \mathrm{~m} \mathrm{x}{ }^{\circ} \mathrm{K}$ ] appearing in eq. 18 were determined from the methane entropy data. With the knowledge of $d_{0}$ and $d_{1}$ all the parameters in eqs. 28 and 29 except $p_{1}$ and $L_{1}$ can be determined using the equations reported in Table 1 . For simplicity we use the virial equation truncated after its second term and from eq. 30 parameter $p_{1}$ in eq. 28 can be determined by minimizing the differences between values of $c$ from eq. 30 and the experimental values. Value of $p_{1}$ for methane in the temperature range of $90-500 \mathrm{~K}$ and pressure up to 100 bar was determined to be -81 . Therefore, from eq. 28 and Table 1 , the following relation for the second virial coefficient of methane can be derived:

$$
B(T)=13.274 \frac{\ln T}{T}+0.0201-81 / T-2.924 \times 10^{3} / T^{2}-0.1073 \times 10^{6} / T^{3}
$$

where $B$ is in $\mathrm{m}^{3} / \mathrm{kmol}$. Predicted values of $\mathrm{c}$ from eq. 30 using eq. 35 for $B$ are within $0.4 \%$ of experimental values for the 200 data points tested. Eq. 35 can be further simplified into the following form for the gas phase:

$$
\mathrm{B}(\mathrm{T})=28.54 \frac{\ln \mathrm{T}}{\mathrm{T}}-0.0194-165.82 / \mathrm{T}
$$

Predicted values of the velocity of sound from eq. 36 are within $0.47 \%$ of the experimental values. The second virial coefficient for methane from eq. 36 versus the data reported by Dymond and Smith (1969) are shown in Fig. 1. Except at some intermediate temperatures, good agreement exists between eq. 36, which is derived from the velocity of sound, and the experimental data reported by Dymond and Smith. Predicted compressibilities from eq. 36 and Dymond and Smith data are compared with the experimental values in Fig. 2. For over 200 data points eq. 36 gives an average deviation of $0.5 \%$ while Dymond and Smith data produce $Z$ values within $0.8 \%$ of the experimental values reported by Goodwin (1974).

The second virial coefficients in the form of Eq. 36 were also determined for ethane and propane from the data on the velocity of sound. The results as well as the data sources are given in Table 2.

The Carnahan-Starling modified version of the Lennard-Jones equation of state (eq. 34) is used to obtain the Lennard-Jones parameters from 
the velocity of sound data as it was discussed earlier in this report. Values obtained for these parameters from the velocity of sound as well as those obtained from the viscosity and the second virial coefficient reported by Hirschfelder et al. (1964) for methane, ethane and propane are reported in Table 3. Absolute average deviations for estimating compressibility factors from the Lennard-Jones parameters are also reported in Table 3.

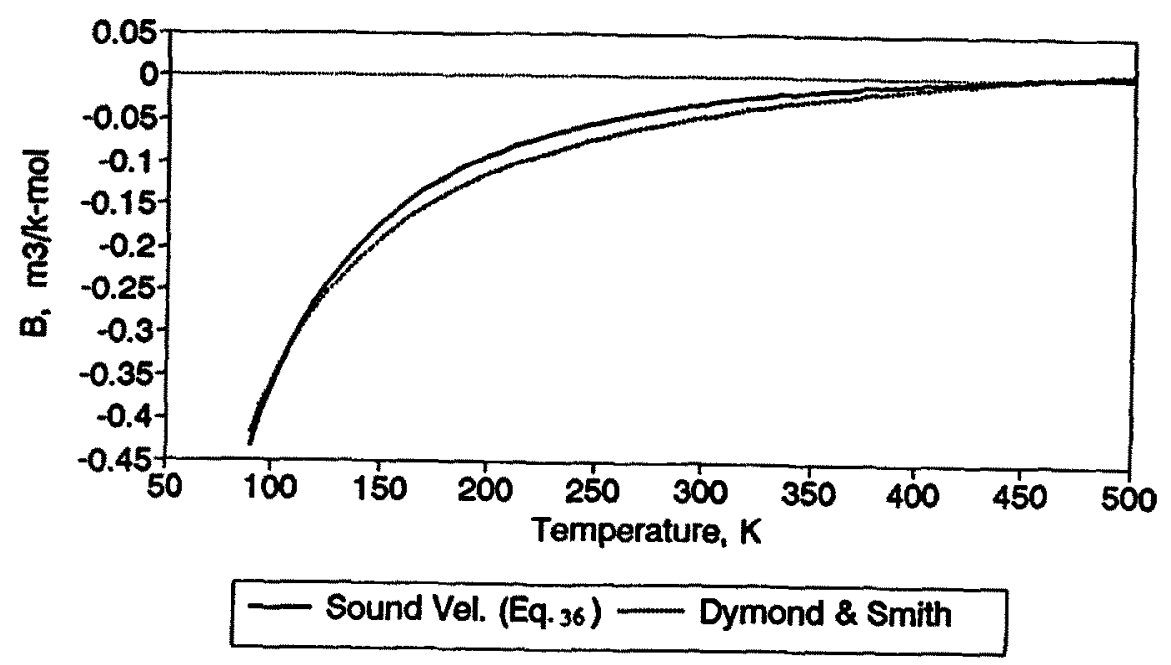

Fig. 1. Second Virial Coefficient for Methane.

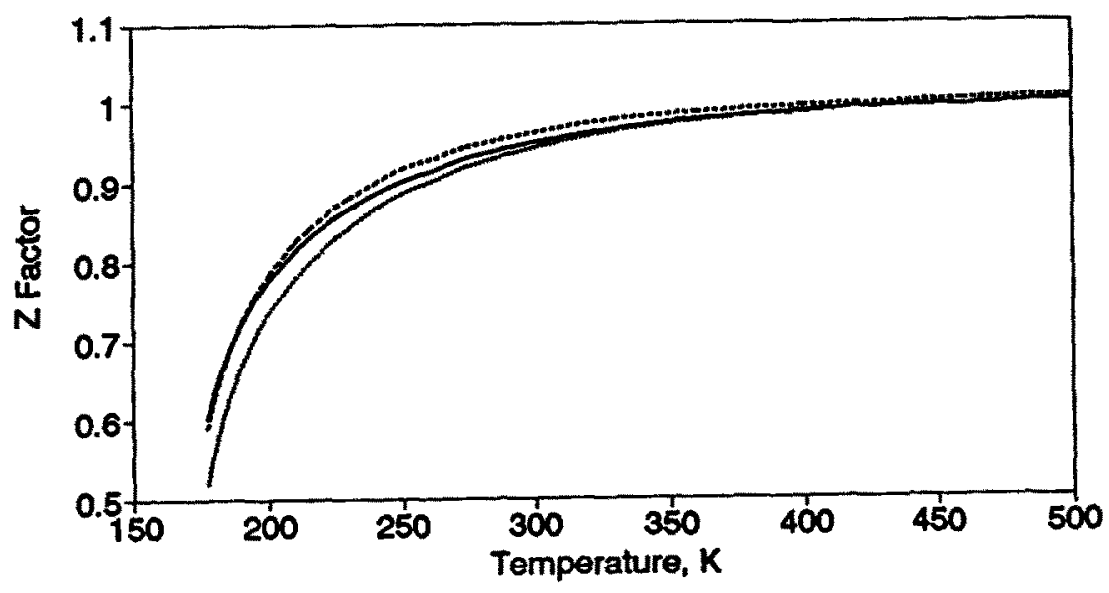

Experimental

- Dymond \& Smith Sound Velocity

Fig. 2. Compressibility Factor for Methane at 30 Bar. 
A graphical comparison for propane at 30 bar is shown in Figure 3.

Data on the velocity of sound for methane, ethane and propane were also used to obtain the van der Waals (vdW) constants. These constants as well as the vdW constants obtained from the critical properties are given in Table 4. Accuracy of constants on prediction of compressibility factors $(Z)$ is also shown in Table 4. For methane and ethane, graphical comparisons for prediction of $Z$ from van der Waals EOS are shown in Figures 4 and 5 , respectively. We believe the larger deviation for $Z$ prediction shown in Fig. 5 is basically due to the inaccuracies of the van der Waals equation of state.

Results obtained for the virial coefficient, the Lennard-Jones potential parameters andthe van der Waals constants show that it is possible to obtain PVT data from cVT data with reasonable degree of accuracy. However, as mentioned earlier, the major goal of this work was to see how the measurable velocity of sound data can be used to obtain the PVT relations. This work does not indicate that the velocity of sound is merely the only appropriate property for such a purpose. There are some other properties, such as the refractive index, which could also be used to obtain parameters of the equations of state.

\section{TABLE 2}

The Second Virial Coefficient Calculations from the Velocity of Sound Data $\mathrm{B}\left[\mathrm{m}^{3} / \mathrm{Kmole}\right]=\mathrm{a} \ln \mathrm{T} / \mathrm{T}+\mathrm{b}+\mathrm{c} / \mathrm{T} ; \mathrm{T}\left[\mathbf{=}^{\circ} \mathrm{K}\right]$

\begin{tabular}{lcccc}
\hline Compound & $\mathrm{a}$ & $\mathrm{b}$ & $\mathrm{c}$ & \% AAD for Z \\
\hline Methane & 28.54 & -0.0194 & -165.82 & 0.5 \\
Ethane & 160.0 & -0.25 & -880.0 & 1.1 \\
Propane & 220.0 & -0.23 & -1290.0 & 1.4
\end{tabular}

Number of Data Points used: 150 for each compound (Goodwin, 1974; Goodwin, et al., 1976; Goodwin and Haynes, 1982). Pressure Range: 0.01-20 MPa (0.1 - 206 bar); Temperature Range: From saturation temperature up to $500^{\circ} \mathrm{K}$ for methane, up to $600 \mathrm{~K}$ for ethane and up to $700 \mathrm{~K}$ for propane.

\section{CONCLUSIONS}

Based on the statistical and classical thermodynamics, coefficients of the equations of state such as the virial coefficients, the Lennard-Jones parameters, the van der Waals constants, etc. can be determined from the velocity of sound data. A generalized cVT relation (eq. 15) is derived which can be used for this purpose. In addition, a new temperature-dependent correlation in the form of eq. 35 is developed for the virial coefficients. In the present report for the demonstration of the technique and due to the availability of both experimental PVT and cVT data, only a few light 
substances are examined. However, the proposed technique is applicable for heavy, complex or newly designed molecules for which the velocity of sound measurements are readily accessible, but their PVT relations may not be available.

\section{TABLE 3}

The Lennard-Jones Potential Parameters from the Velocity of Sound Data and Other Sources
Source of Data
$\epsilon / \mathbf{k}_{\mathbf{B}}\left({ }^{\bullet} \mathrm{K}\right)$
o ('A)
$\% A A D$ for $\mathbf{Z}$

Methane:

Velocity of Sound

178.1

Second Virial Coefficient

148.2

3.97

0.8

Viscosity

144.0

3.817

4.0

Ethane:

Velocity of Sound

300.0

3.796

4.7

Second Virial Coefficient

243.0

4.25

0.5

Viscosity

230.0

3.954

3.0

Propane:

Velocity of Sound

Second Virial Coefficient

Viscosity
350.0

242.0

254.0
4.418

3.4

5.0

5.637

5.061
1.1

11.5

8.0

Data sources for the velocity of sound and PVT data and the temperature and pressure ranges are given in Table 2. The LJ parameters are based on the second virial coefficient and viscosity data and are taken from Hirschfelder, et al. (1964). $1^{\circ} \mathrm{A}=10^{-10} \mathrm{~m}$.

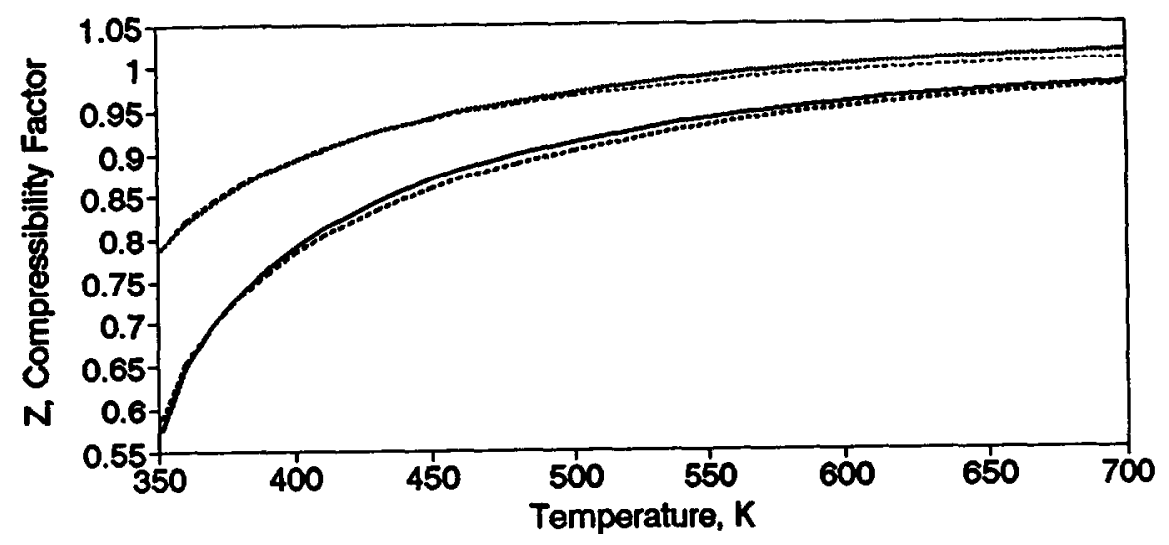

- Exp. $\quad$........ Sound Vel. — Virial Coeff. …… Viscosity

Fig. 3. Compressibility Factor for Modified LJ EOS for Propane at 30 Bar. 


\section{TABLE 4}

The van der Waals Constants from the Velocity of Sound and the Critical Properties Data*

\begin{tabular}{lccc}
\hline Compounds & $\mathrm{a}$ & $\mathrm{b}$ & $\%$ AAD for $Z$ \\
& $\mathrm{~m}^{6} /\left(\mathrm{Kmole}^{2} . \mathrm{MPa}\right)$ & $\mathrm{m}^{3} / \mathrm{Kmole}$ &
\end{tabular}

Methane:

Velocity of Sound
Original Constants

0.188583

0.04478

1.0

0.227209

0.04305

0.8

Ethane:

Velocity of Sound
Original Constants

0.384613

0.549447

0.05718
0.05198

1.8

0.05198

2.4

Propane:

Velocity of Sound

Original Constants

0.834060

0.09051

0.926734

0.09051

1.4

1.5

Data sources and the Pressure and temperature ranges are given in Table 2. The LJ parameters are based on the second virial coefficient and viscosity data and are taken from Hirschfelder, et al. (1964). Original constants for the vdW equation of state were determined through the use of the critical temperature and pressure.

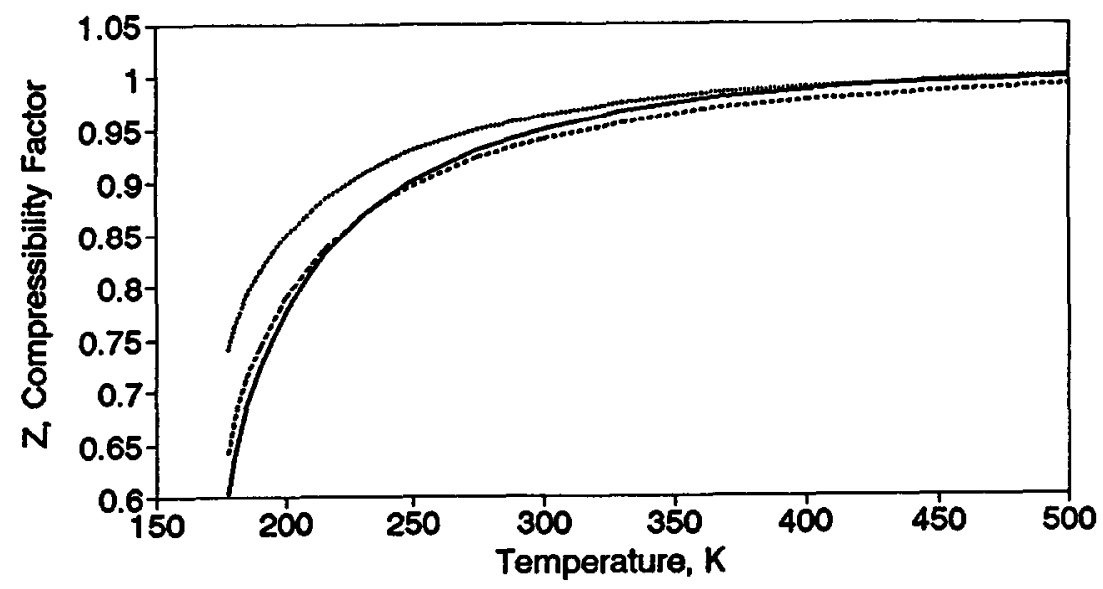

Experimental

Sound Vel.

......... Original voW Const

Fig. 4. Compressibility Factor from van der Waals EOS for Methane at 30 Bar. 


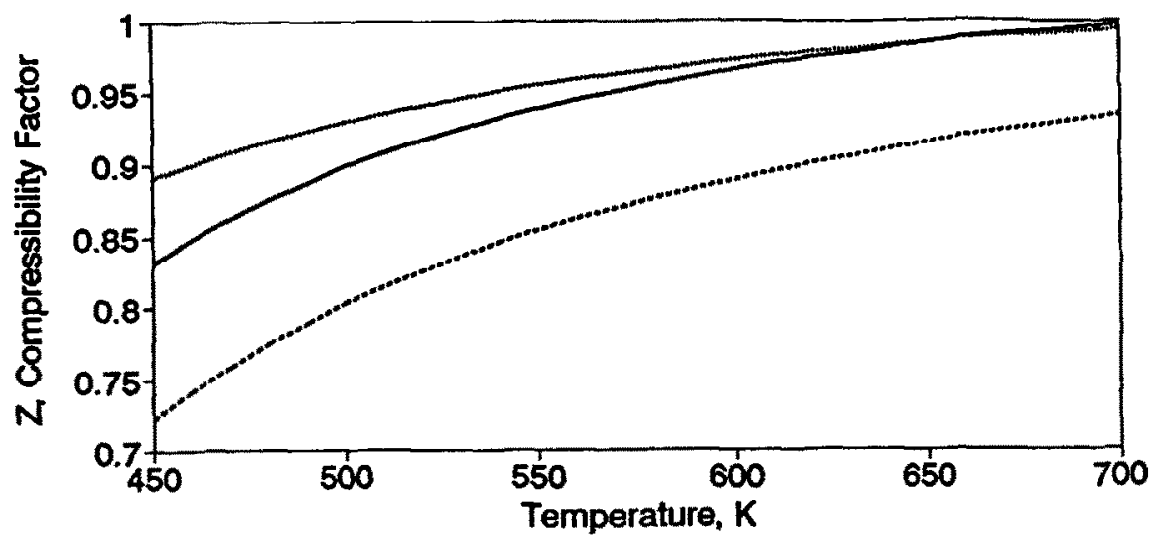

- Experimental $=$ Sound Vel. -....... Original Const.

Fig. 5. Compressibility Factor from van der Waals EOS for Ethane at 100 Bar.

ACKNOWLEDGEMENT: This research is supported by the National Science Foundation Grant No. CTS-9108595.

\section{REFERENCES:}

Alem, A.H. and Mansoori, G.A., 1984. The VIM Theory of Molecular Thermodynamics. AIChE J, 30: 468-480.

Chorn, L.G. and Mansoori, G.A. (Editors), 1988. $\mathrm{C}_{7}+$ Fraction Characterization, Advances in Thermodynamics, Vol. I, Taylor \& Francis Pub. Co., Bristol, PA.

Colgate, S.O., Silvarman, A. and Dejsupa, C., 1992. Acoustic resonance determination of sonic speed and the critical point. AIChE Spring Meeting, paper No. 29c, New Orleans.

Dymond, J.H. and Smith, E.B., 1969. The Virial Coefficient of Gases, A Critical Compilation. Oxford Univ. Press, Oxford, GB.

Edalat, M, Bozar-Jomehri, R.B. and Mansoori, G.A., 1993. Generalized Equation predicts vapor pressure of Hydrocarbons. Oil \& Gas Journal, February 1: 39-40.

Goodwin, R.D., 1974. The Thermophysical Properties of Methane, from 90 to $500 \mathrm{~K}$ at Pressures to 700 Bar. National Bureau of Standards, NBS Tech. Note 653, April.

Goodwin, R.D., Roder, H.M. and Straty, G.C., 1976. Thermophysical Properties of Ethane from 90 to $600 \mathrm{~K}$ at Pressures to 700 Bar. National Bureau of Standards, NBS Tech. Note 684, August.

Goodwin, R.D. and Haynes, W.M., 1982. Thermophysical Properties of Propane from 85 to $700 \mathrm{~K}$ at Pressures to $70 \mathrm{MPa}$. National Bureau of Standards, NBS Monograph 170, April.

Hirschfelder, J.O., Curtiss, C.F. and Bird, R.B., 1964. Molecular Theory of Gases and 
Liquids. John Wiley \& Sons, Inc., New York, NY.

Rao, R.V.G. and Dutta, S.K., 1983. An equation of state of L-J fluids and evaluation of some thermodynamic properties. Z. Phys. Chem. 264: 771-783.

Rao, R.V.G. and Gupta, B.D., 1985. Visco-elastic properties of ionic liquids. Acoustics Letters, 8: 9, 147-149.

Prausnitz, J.M., Lichtenthaler, R.N. and de Azevedo, E.G., 1986. Molecular Thermodynamics of Fluid Phase Equilibria (2nd Edition). Prentice-Hall, Englewood Cliffs, N.J.

Walas, S.M., 1985. Phase Equilibria in Chemical Engineering,. Butterworth Publishers, London, GB.

Whitson, C.H., 1984. Effect of physical properties estimation on equation-of-state predictions, SPEJ, December: 685-696.

\section{NOMENCLATURE:}

\begin{tabular}{|c|c|c|}
\hline B & $=$ & Second virial coefficient \\
\hline $\mathbf{B}^{\prime}$ & $=$ & $\mathrm{dB} / \mathrm{dT}=$ First derivative of $\mathrm{B}$ with respect to temperature \\
\hline B" & $=$ & $d^{2} B / d T^{2}=$ Second derivative of $B$ with respect to $T$ \\
\hline C & $=$ & Third virial coefficient \\
\hline$C^{\prime}$ & $=$ & $\mathrm{dC} / \mathrm{dT}=$ First derivative of $\mathrm{C}$ with respect to temperature \\
\hline c & $=$ & Velocity of sound \\
\hline$c_{p}$ & $=$ & Constant pressure heat capacity \\
\hline $\mathrm{C}_{\mathbf{v}}$ & $=$ & Constant volume heat capacity \\
\hline d & $=$ & hard-sphere diameter \\
\hline$d_{0} \& d_{1}$ & $=$ & Constants in eq. 18 \\
\hline $\mathbf{k}_{\mathrm{B}}$ & $=$ & Boltzman constant \\
\hline$L_{n}$ & $=$ & Constant in eq. 29 \\
\hline $\mathbf{M}$ & $=$ & Molecular weight \\
\hline $\mathbf{N}_{\mathbf{a}}$ & $=$ & Avogadro number \\
\hline $\mathbf{p}$ & $=$ & Pressure \\
\hline$P_{\mathbf{n}}$ & $=$ & Constants in eq. 28 \\
\hline $\mathbf{q}_{\mathbf{n}}$ & $=$ & Constants in eqs. $28 \& 29$ \\
\hline $\mathbf{R}$ & $=$ & Gas constant \\
\hline $\mathbf{s}$ & $=$ & Entropy \\
\hline $\mathbf{T}$ & $=$ & Temperature \\
\hline $\mathbf{v}$ & $=$ & Molar volume \\
\hline $\mathbf{Z}$ & $=$ & Compressibility factor \\
\hline \multicolumn{3}{|c|}{ Greek Letters: } \\
\hline$\sigma$ & $=$ & Size parameter in the Lennard-Jones potential \\
\hline $\boldsymbol{E}$ & $=$ & Energy parameter in the Lennard-Jones potential \\
\hline$\rho$ & $=$ & Molar density \\
\hline $\boldsymbol{\gamma}$ & $=$ & Heat capacity ratio \\
\hline $\boldsymbol{\kappa}_{\mathbf{T}}$ & $=$ & Isothermal compressibility \\
\hline$\eta$ & $=$ & Packing fraction \\
\hline \multicolumn{3}{|c|}{ Superscripts: } \\
\hline id & $=$ & Ideal gas reference state of unit pressure \\
\hline \multicolumn{3}{|c|}{ Subscript: } \\
\hline hs & $=$ & hard-sphere \\
\hline
\end{tabular}

